\title{
DESEMPEÑO SEXUAL DE MACHOS DE LABORATORIO ESTÉRILES DE ANASTREPHA OBLIQUA ACLIMATADOS BAJO CONDICIONES DE CAMPO
}

\author{
José Luis Quintero Fong, María del Refugio Hernández IbarRa \\ y Dina Orozco Dávila \\ Programa Operativo Moscafrut. Central Poniente No. 14 altos-Esq. 2a . Avenida Sur. CP 30700. \\ Tapachula, Chiapas, México. \\ e-mail: quinterofong@hotmail.com
}

Quintero-Fong, J. L., M. R. Hernández-Ibarra \& D. Orozco Dávila. 2011. Desempeño sexual de machos de laboratorio estériles de Anastrepha obliqua aclimatados bajo condiciones de campo. Acta Zool. Mex. (n. s.), 27(1): 17-23.

RESUMEN. Se evaluó por medio de pruebas estándar de control de calidad el desempeño sexual de machos de cría estériles de Anastrepha obliqua aclimatados bajo condiciones de campo. Machos estériles de cría de laboratorio fueron mantenidos en un huerto de mango en jaulas de madera durante 0,2 , 4, 6 y 8 días antes de competir con machos silvestres por hembras silvestres en pruebas de desempeño sexual. Los machos de laboratorio fueron evaluados con 8 días de edad mientras los silvestres con 15 días. Este estudio fue conducido para confirmar si los insectos mantenidos lo más cerca posible a las condiciones naturales, obtienen una ventaja en su desempeño sexual con respecto a machos mantenidos en condiciones artificiales. Los resultados del estudio indicaron que la proporción de apareamientos en la prueba fue satisfactoria y los valores del índice de esterilidad relativa no muestran diferencias significativas entre los distintos tratamientos. En conclusión los datos indican que el pre-acondicionamiento de machos de A. obliqua a condiciones de campo no confiere una ventaja en el desempeño sexual con hembras silvestres en jaulas de campo, lo cual es ideal para los programas de liberación donde la técnica del insecto estéril es aplicada.

Palabras clave: A. obliqua, cría masiva, aclimatación, desempeño sexual.

Quintero-Fong, J. L., M. R. Hernández-Ibarra \& D. Orozco Dávila. 2011. Sexual performance of laboratory sterile Anastrepha obliqua males acclimated under field conditions. Acta Zool. Mex. (n. s.), 27(1): 17-23.

ABSTRACT. Standard quality control tests were carried out to evaluate the sexual performance of laboratory sterile Anastrepha obliqua males acclimated under field conditions. Laboratory sterile males were maintained in a mango orchard in wooden cages for $0,2,4,6$ and 8 days before competing with wild males for matings with wild females in sexual performance tests. Laboratory males were evaluated at 8 days of age while wild males were 15 days old. This study was conducted to confirm whether insects kept as close as possible to natural conditions, obtain an advantage in their sexual performance

Recibido: 13/10/2009; aceptado: 02/09/2010. 
with respect to males maintained under artificial conditions. The results of the study indicated that the mating proportion in the test was within SIT quality control standards and the relative sterility index values didn't show significant differences between the different treatments. In conclusion the data indicates that the pre-conditioning of A. obliqua males to field conditions doesn't give an advantage over non-conditioned males in sexual performance with wild females in field cages.

Key words: A. obliqua, rearing-mass, acclimation, sexual performance

\section{INTRODUCCIÓN}

Anastrepha obliqua (Macquart) (Diptera: Tephritidae) es considerada la plaga del mango (Mangifera indica L.) de mayor importancia en México (Aluja 1994). Esta especie, comúnmente conocida como mosca de la fruta de las indias occidentales, es endémica en el centro y sur de América y es probablemente una de las especies dentro del género Anastrepha de la familia Tephritidae más ampliamente distribuida. En México, es una plaga importante de mango y ciruelo tropical (Spondias spp.) (Hernández-Ortiz y Aluja 1993). Esto resulta estrictas restricciones cuarentenarias en los Estados Unidos de Norte América, el principal importador del mango Mexicano (APHIS 1994). Debido a su impacto económico y a la amenaza que representa para la industria frutícola, en el año 2001 se implementó la aplicación de la técnica del insecto estéril (TIE) contra esta plaga en diferentes estados del norte de México (Orozco et al. 2004). Sin embargo, a pesar de su éxito e incrementado uso, existe la necesidad de mejorar este método. Esta técnica, requiere producir grandes cantidades de insectos (a un costo razonable) sin afectar la habilidad de los insectos de exhibir un comportamiento que permita que los machos estériles compitan con los machos silvestres y transfieran su esperma a hembras silvestres. Esto puede ser evaluado periódicamente mediante el monitoreo del comportamiento sexual por medio de pruebas de control de calidad (Boller et al. 1981). La compatibilidad (aceptación de individuos de una población por individuos del otro sexo de otra población) y competitividad (éxito de apareamiento del macho estéril en competencia con machos silvestres) fueron en el pasado pruebas realizadas en el laboratorio (Fried 1971). Sin embargo, las pruebas de laboratorio no evalúan totalmente el comportamiento de los insectos ya que las condiciones son demasiado artificiales y no siempre se produce el repertorio completo como para hacer una evaluación certera por lo que se hace necesario realizar pruebas de campo (Robinson et al. 2002; Meza y Díaz 2006). Actualmente las pruebas de desempeño sexual se realizan en jaulas de campo que contienen un árbol de tamaño pequeño en su interior siguiendo protocolos establecidos que permiten estimar índices de desempeño los cuales luego pueden ser comparados con valores estándar (FAO/IAEA/USDA 2003). En algunas especies, para un mejor desempeño sexual, los insectos de cría son previamente aclimatados a campo. Si bien para A. ludens (Meza y Díaz 2006) y A. obliqua (Meza et al. 2002) se reporta una aclimatación de dos días antes de la prueba, existe escasa información sobre el efecto de esta medida, 
mientras que para Ceratitis capitata (Wiedemann) pruebas preliminares reportan que la aclimatación de machos a 0,1 y 3 días no confiere ventaja en su comportamiento sexual (Pereira et al. 2007). En esta investigación evaluamos el desempeño sexual de machos de cría estériles de $A$. obliqua mantenidos bajo condiciones de campo durante varios periodos de tiempo. Este estudio fue conducido para confirmar si los insectos de cría estériles mantenidos en condiciones lo más cerca posible a las naturales, poseen una ventaja en su desempeño sexual frente a insectos mantenidos en condiciones artificiales.

\section{MATERIALES Y MÉTODOS}

Sitio de estudio. Las pruebas fueron realizadas en un huerto de mango cv. Ataulfo $\left(14^{\circ} 55^{\prime}\right.$ " 08.9" N, 92 $16^{\prime} 34.2^{\prime \prime} \mathrm{W}$, y altitud $137 \mathrm{~m}$ ) cerca de Tapachula, Chiapas, México.

Insectos. Las moscas silvestres fueron obtenidas de frutos infestados de Spondias mombin L. (Anacardiaceae) colectados en la región del Soconusco, Chiapas, México. El manejo de la larva y pupa colectada fue similar al descrito previamente por Aluja et al. (1997). Las moscas de cría estériles fueron obtenidas de pupas producidas en la Planta Moscafrut de Metapa de Domínguez, Chiapas, México. Pupas de 13 días de edad (48 horas antes de la emergencia) fueron irradiadas a 80 Gy en una fuente de cobalto 60 instalada en un equipo irradiador modelo Gamma beam 127 (GB-127). Esta dosis produce $100 \%$ de esterilidad en los machos de $A$. obliqua. Las pupas de ambas cepas fueron colocados en jaulas de madera de $30 \times 30 \times 30 \mathrm{~cm}$ cubiertas con malla tull (de $2 \mathrm{~mm}$ ) para su emergencia. Las moscas emergidas fueron separadas por sexo y mantenidas en cuartos separados para evitar cualquier contacto con feromonas sexuales antes de la prueba. Todas las moscas fueron alimentadas con agua y una mezcla de proteína hidrolizada (ICN Biomedical, Aurora, OH): azúcar (azúcar de caña estándar marca grupo porres) (1:3).

Aclimatación en campo. Los insectos de cría estériles antes de la prueba fueron sometidos bajo condiciones de campo en jaulas de madera de $30 \times 30 \times 30 \mathrm{~cm}$ a 5 diferentes tratamientos: (1) cero días de aclimatación, (2) dos días de aclimatación, (3) cuatro días de aclimatación, (4) seis días de aclimatación y (5) ocho días de aclimatación. Los insectos fueron mantenidos con agua y alimento (proteína hidrolizada y azúcar) sin riesgo de depredación y sin exposición a lluvias. Los insectos de cría (sin aclimatación o previo a la aclimatación) y silvestres fueron mantenidos a condiciones de laboratorio a $25 \pm 1{ }^{\circ} \mathrm{C}, 65 \pm 5 \%$ de humedad relativa y mantenidos bajo un fotoperiodo de 12 horas luz (de 7:00 a 19:00 horas): 12 horas oscuridad hasta el día de la prueba.

Desempeño sexual. Las pruebas para evaluar el desempeño sexual de los machos estériles se realizaron entre insectos de cría (de cada uno de los tratamientos) y sil- 
vestres. Las jaulas de campo, la preparación del material y el marcaje de los insectos, fue realizado siguiendo los métodos utilizados por Meza-Hernández y Díaz-Fleischer (2006). Para ello, se liberaron 40 parejas de moscas vírgenes en cada jaula (20 parejas de cada cepa). Las moscas de cría fueron evaluadas con 8 días de edad y los silvestres con 15 días de edad. El número de apareamientos fue registrado en intervalos de 30 min. Se evaluaron siete jaulas por tratamiento usando diferentes cohortes de insectos (una replica por cohorte). Las evaluaciones fueron realizadas de 06:00 a 12:00 h, intervalo que comprende el período de máxima actividad sexual para esta especie (Aluja et al. 2000).

Análisis estadístico. Las condiciones del ensayo fueron evaluadas por medio de la proporción de apareamientos (siglas en inglés PM) (FAO/IAEA/USDA 2003) mientras que el desempeño de los machos estériles se midió con el índice de esterilidad relativa (siglas en inglés RSI) (McInnis et al. 1996). La PM mide la capacidad de apareamiento de las moscas y representa el total de la actividad sexual. Para que una réplica sea considerada en el análisis el valor de $\mathrm{PM}$ debe ser mayor a $0.2(\mathrm{FAO} /$ IAEA/USDA 2003).

$$
\mathrm{PM}=\frac{\text { Número de parejas colectadas }}{\text { Número de hembras liberadas }}
$$

El RSI mide la proporción de apareamientos de hembras silvestres con machos estériles. Bajo condiciones donde el número de machos estériles y silvestres liberados es igual, valores de RSI mayores a 0.50 indican alta competitividad de los machos estériles mientras que valores menores a 0.50 indican baja competitividad de los mismos (McInnis et al. 1996). Valores cercanos a 0.50 indican igual desempeño.

$$
\begin{aligned}
& \text { Apareamiento macho-hembra } \\
& \mathrm{C}=\text { Insectos de cría estériles } \\
& \mathrm{S}=\text { Insectos silvestres }
\end{aligned}
$$

Los datos fueron analizados por medio de un análisis de varianza (Ott y Longnecker 2001). El valor de significancia usado en esta prueba fue de 95\%. El análisis estadístico fue realizado con el software StatView ver. 5.0.

\section{RESULTADOS}

En todas las jaulas de campo se registraron valores de PM mayores a 0.25 (más del $25 \%$ de las hembras se aparearon) por lo que todas las repeticiones fueron consideradas en el análisis (FAO/IAEA/USDA 2003). Los resultados de PM y RSI se presentan en el Cuadro 1. No se encontraron diferencias significativas entre tratamientos tanto para PM $\left(\mathrm{F}_{4.30}=0.55 ; \mathrm{P}=0.70\right)$ como para $\mathrm{RSI}\left(\mathrm{F}_{4.30}=0.080 ; \mathrm{P}=0.98\right)$. Sin embargo, los valores del RSI en cada uno de los tratamientos indicaron que los machos silvestres exhibieron un mejor desempeño sexual que los machos de cría estériles. 
Cuadro 1. Proporción de apareamientos (PM) e índice de esterilidad relativa (RSI) de machos de $A$. obliqua con diferentes días de aclimatación a condiciones de campo.

\begin{tabular}{ccc}
\hline Días de aclimatación & PM $( \pm$ SE $)$ & RSI $( \pm$ SE $)$ \\
\hline 0 & $0.38 \pm 0.03 \mathrm{a}$ & $0.36 \pm 0.12 \mathrm{a}$ \\
2 & $0.38 \pm 0.05 \mathrm{a}$ & $0.37 \pm 0.07 \mathrm{a}$ \\
4 & $0.36 \pm 0.04 \mathrm{a}$ & $0.35 \pm 0.10 \mathrm{a}$ \\
6 & $0.44 \pm 0.05 \mathrm{a}$ & $0.30 \pm 0.10 \mathrm{a}$ \\
8 & $0.37 \pm 0.04 \mathrm{a}$ & $0.32 \pm 0.14 \mathrm{a}$ \\
\hline
\end{tabular}

Media \pm error estándar con igual letra en una columna no indican diferencias significativas $(p \geq 0.05)$.

\section{DISCUSIÓN}

Los resultados del estudio indicaron que el pre-acondicionamiento en campo de machos de cría estériles de $A$. obliqua, no confiere una ventaja en el desempeño sexual frente a hembras silvestres en jaulas de campo. Estos resultados son similares a los obtenidos en C. capitata, donde machos de cría mantenidos por 0,1 y 3 días que no mostraron un incremento en su desempeño sexual (Pereira et al. 2007). Sin embargo, en esta investigación se menciono la necesidad de estudiar periodos de tiempo más largos como los evaluados en este estudio. En nuestras evaluaciones los machos de cría de $A$. obliqua fueron mantenidos por $0,2,4,6$ y 8 días en condiciones de campo antes de competir con insectos silvestres. Sin embargo, no se presentaron diferencias significativas en la proporción de apareamientos con hembras silvestres. Estos resultados sugieren que el pre-acondicionar a los insectos de cría para pruebas de calidad en jaulas de campo, no tiene ningún efecto en el desempeño sexual de $A$. obliqua. Otro aspecto importante para el éxito de la TIE son las condiciones en que son mantenidos los insectos antes de su liberación en el campo para permitir la emergencia y maduración sexual. El tiempo requerido varia entre especies, para $C$. capitata se reportan de 4 a 7 días, para A. ludens de 5 a 7 días y para A. obliqua 5 días (SAGARPA 1999; Tirado y Gómez-Escobar 2005). Si bien este tiempo puede ser utilizado para brindar a los machos condiciones que favorezcan su competitividad en el campo como son la exposición a volátiles que les confieren mayor competitividad sexual (Shelly et al. 2007) o una dieta con proteínas (Yuval et al. 2007), es cierto que periodos largos pueden ser contraproducentes ya que se necesita espacio para mantener a las moscas y se incrementa la mortalidad por lo que se intenta reducir dichos tiempos mediante el uso de aceleradores del proceso de maduración sexual como es el metropeno, análogo de la hormona juvenil (Faria et al. 2008). Este estudio sostiene la idea de que el tiempo de permanencia de los insectos bajo estas condiciones no afecta su comportamiento sexual y es probable que cualquier efecto se origine del manejo durante el empacado y liberado de los insectos. Sin embargo, a fines de establecer claramente el impacto de cada uno de estos factores y otros relacionados a la cría que puedan estar dismi- 
nuyendo la competitividad de los machos estériles, es necesario realizar estudios con insectos sometidos a estos procesos.

En conclusión los resultados de este estudio indican que el pre-acondicionamiento en campo de machos de cría de A. obliqua antes de su liberación no les confiere una ventaja en el desempeño sexual frente a hembras silvestres. Esto resulta beneficioso para los programas de liberación ya que no se hace necesario la implementación de un sistema de aclimatación previo a las liberaciones.

AGRADECIMIENTOS. Se agradece al grupo de Validación Tecnológica de la Planta Moscafrut: Marco P. Pérez, José L. Zamora y Jesús A. Escobar por su soporte técnico durante la planificación y desarrollo del proyecto. Esta investigación fue financiada por el Programa Operativo Moscafrut.

\section{LITERATURA CITADA}

Aluja, M. 1994. Bionomics and management of Anastrepha. Annual Review of Entomology, 39: 155-178.

Aluja, M., A. Jiménez, J. Piñero, M. Camino, L. Aldana, M. E. Valdés, V. Castrejón, I. Jácome, A. B. Dávila \& R. Figueroa. 1997. Daily activity patterns and within-field distribution of papaya fruit flies (Diptera: Tephritidae) in Morelos and Veracruz, México. Annals of the Entomological Society of America, 90: 505-520.

Aluja, M., J. Piñero, I. Jácome, F. Díaz-Fleischer \& J. Sivinski. 2000. Behavior of flies in the genus Anastrepha (Trypetinae: Toxotrypanini), pp. 375-406. In: M. Aluja \& A. Norrbom (Eds.). Fruit Flies (Tephritidae): Phylogeny and Evolution of Behavior. CRC Press, Boca Raton, FL, USA.

[APHIS] Animal and Plant Health Inspection Service. 1994. Plant protection and quarantine treatment manual. United States Department of Agriculture. (APHIS-PPQ) Section IV. TI02 (c). Mango.

Boller, E. F., B. I. Katsoyannos, U. Remund \& D. L. Chambers. 1981. Measuring, monitoring and improving the quality of mass-reared Mediterranean fruit flies, Ceratitis capitata (Wied.). 1. The RAPID quality control system for early warning. Zeitschrift für Angewandte Entomologie, 92: 67-83.

FAO/IAEA/USDA. 2003. Manual for Product Quality Control and Shipping Procedures for Sterile Mass-Reared Tephritid Fruit Flies, Version 5.0. International Atomic Energy Agency, Vienna, Austria, $85 \mathrm{pp}$.

Faria, M. J., R. Pereira, T. Dellinger \& P. E. A. Teal. 2008. Influence of methoprene and protein on survival, maturation and sexual performance of male Ceratitis capitata (Diptera:Tephritidae). Journal of Applied Entomology, 132: 812-819.

Fried, M. 1971. Determination of sterile insect competitiveness. Journal of Economic Entomology, 64: 869-872.

Hernández-Ortiz, V. \& M. Aluja. 1993. Listado de especies del genero neotropical Anastrepha (Diptera: Tephritidae) con notas sobre su distribución y plantas hospederas. Folia Entomológica Mexicana, 88: 89-105.

McInnis, D. O., D. R. Lance \& C. G. Jackson. 1996. Behavioral resistance to the sterile insect release technique by the Mediterranean fruit fly (Diptera: Tephtritidae) in Hawaii. Annals of the Entomological Society of America, 89: 739-744.

Meza, J. S., E. Hernández, M. Salvador-Figueroa \& L. Cruz-López. 2002. Sexual compatibility, mating performance and sex pheromone release of mass-reared and wild Anastrepha obliqua (Diptera: 
Tephritidae) under field-cage conditions, pp. 99-104. In: B. N. Barnes (Ed.) Proceeeding of $6^{\text {th }}$ International symposium, 6-10 May 2002, Isteg Scientific Publications, Stellenbosch, South Africa.

Meza-Hernández, J. S. \& F. Díaz-Fleisher. 2006. Comparison of sexual compatibility between laboratory and wild Mexican fruit flies under laboratory and field conditions. Journal of Economic Entomology, 99: 1979-1986.

Orozco, D., J. Domínguez, J. Reyes, A. Villaseñor \& J. M. Gutiérrez. 2004. SIT and biological control of Anastrepha fruit flies in Mexico, pp. 245-249. In: B. N. Barnes (Ed.) Proceedings of $6^{\text {th }}$ International Symposium, 6-10 May 2002, Isteg Scientific Publications, Stellenbosch, South Africa.

Ott, R. L. \& M. Longneaker. 2001. An Introduction of Statistics Methods and Data Analyses (5 ${ }^{\text {th }}$ ed.). Duxbury Publishers, Pacific Grove, California, USA.

Pereira, R., N. Silva, C. Quintal, R. Abreu, J. Andrade \& L. Dantas. 2007. Effect of acclimation to outdoor conditions on the sexual performance of mass-reared medflies (Diptera: Tephritidae). Florida Entomologist, 90: 171-174.

Robinson, A. S., J. P. Cayol \& J. Hendrichs. 2002. Recent findings on medfly sexual behavior: implications for SIT. Florida Entomologist, 85: 171-181.

SAGARPA. 1999. Norma Oficial Mexicana NOM-023-FITO-1995. Apéndice técnico para las operaciones de campo de la campaña nacional contra moscas de la fruta. Febrero 1999, Gobierno de México.

Shelly T. E., D. McInnis, C. Rodd, J. Edu \& E. Pahio. 2007. Sterile Insect Technique and Mediterranean Fruit Fly (Diptera: Tephritidae): Assessing the Utility of Aromatherapy in a Hawaiian Coffee Field. Journal of Economic Entomology, 100: 273-282.

Tirado, P. L \& E. Gómez-Escobar. 2005. Prácticas de empaque y liberación de moscas y parasitoides. Anexo 3, 3 p. In: Memorias del XVI curso internacional sobre moscas de la fruta. Metapa de Domínguez, Chiapas, México.

Yuval, B., K. Levy, R. Kaspi, P. Taylor \& T. Shelly. 2007. Breakfast of champions or kiss of death? Survival and sexual performance of protein-fed, sterile Mediterranean fruit flies (Diptera: Tephritidae). Florida Entomologist, 90: 115-122. 